\title{
Prevalence of vitamin D deficiency in adult patients with systemic lupus erythematosus in Kingdom of Bahrain
}

\begin{abstract}
Introduction: Vitamin D deficiency (VDD) has been implicated as an important factor in the development or aggravation of systemic lupus erythematosus (SLE). Patients with SLE are especially prone to the development of VDD due to the nature of their illness and avoidance of sun exposure. However, the prevalence of VD status in Bahraini patients with SLE has not been studied before. Our aim is to study the prevalence of VDD in Bahraini cohort with SLE.
\end{abstract}

Material and methods: Fifty-eight Bahraini patients with SLE were included retrospectively in this study. The mean age of the patients, was 39.78years (16.0 - 61.0, STD 12.98). Most of the patients were females 50/58. The patients were followed at the rheumatology department at "Salmanyia medical complex" in Bahrain. Controls were fifty-eight age-matched healthy Bahraini subjects. Serum levels of $25(\mathrm{OH})$ vitamin D3 were estimated using chemilumenescence immunoassay. Chi Square and $\mathrm{T}$-Test were used for analysis a $\mathrm{p}$ value of $\leq 0.05$ was considered significant.

Results: There was statistically significant difference $(\mathrm{p} \leq 0.05)$ in the mean serum levels of vitamin D between patients $(30.67 \mathrm{nmol} / 1)$ and controls $(39.95 \mathrm{nmom} / \mathrm{L})$. There was a statistically significant difference in Phos, PTH and ALP between patients and control group with a $\mathrm{p} \leq 0.001$ for each. In the SLE patients there were $49.1 \%$ deficient, $45.1 \%$ insufficient and $3.8 \%$ were Optimal. In the controls; $27 \%$ was deficient, $52 \%$ insufficient and $21 \%$ optimal.

Conclusion: There was high prevalence of VDD in Bahraini patients with SLE. Both patients and controls had low vitamin D levels, however the patients had statistically significant lower vitamin D serum levels compared to the controls. Our study also highlights the need for studying the effects of correcting hypovitaminosis on the clinical disease activity in Bahraini SLE patients.

Keywords: vitamin D, SLE, bahrain
Volume 6 Issue I - 2017

\section{Eman Farid,' Adla B Hassan, ${ }^{2}$ Ahmed A Jaradat, ${ }^{3}$ Ola Al- Segai ${ }^{4}$}

'Consultant Immunologist, Department of Pathology, Salmanyia Medical Complex Hospital, Bahrain

${ }^{2}$ Consultant Rheumatologist, Department of Internal Medicine, Arabian Gulf University, Bahrain

${ }^{3}$ Assistant professor, Department of Statistics, Arabian Gulf University, Bahrain

${ }^{4}$ Consultant Biochemistry; Department of Pathology, Salmanyia Medical Complex Hospital, Bahrain

Correspondence: Adla B Hassan, Department of Internal Medicine, College of Medicine and Medical Sciences, Arabian Gulf University, Manama, Bahrain, Tel 00973 39764|45/00973 17239628, Fax +973 17289388,

Email adla.hassan@gmail.com, adlabmh@agu.edu.bh

Received: January 30, 2017 | Published: October 24, 2017
Abbreviations: SLE, systemic lupus erythematous; VDD, vitamin D deficiency; Phos, phosphate; Ca, calcium; ALP, alkaline phosphatase; PTH, parathyroid hormone; Ds-DNA, double strandedDNA; C3, complement 3; C4, complement 4; ANA, antinuclear antibody

\section{Introduction}

Systemic lupus erythematous (SLE) is a multi-systemic autoimmune rheumatic disease, where environmental and genetic factors are reported to play a major role in the pathogenesis of the disease. ${ }^{1}$ Low vitamin $\mathrm{D}$ status is considered one of these factors. ${ }^{2}$ Usually, exposure to UVB light triggers the endogenous production of vitamin D in the skin and consequently will be converted to the metabolically active form in the kidney. In SLE patient's photosensitivity is considered as a risk factor that could precipitate a flare up and aggravate disease activity. ${ }^{3}$ Therefore, use of sun protection is a keystone in maintaining remission in these patients. However, avoidance of sun exposure may diminish vitamin $\mathrm{D}$ synthesis and aggravated Vitamin D deficiency (VDD). ${ }^{4} \mathrm{~A}$ part from photosensitivity and the use of sun protection, there are other factors that may place SLE patients at increased risk of VDD such as; renal involvement ${ }^{5-7}$ and use of medications for example; anticonvulsants, antimalarial, calcineurin inhibitors or glucocorticoids, that either alter the metabolism of vitamin D or down-regulate the functions of its receptors. ${ }^{8}$ A mechanism other than photo- protection that could intensify VDD in SLE patients is the production of autoantibodies directed against of vitamin D.

Despite the evidence that many studies in different populations report a high prevalence of VDD among patients with SLE, it still remains under-recognized and under-treated in patients with SLE disease. A retrospective longitudinal study of Italian adult SLE patients showed that Vitamin D insufficiency detected in the wintertime can be either a predisposing factor for flare or the consequence of the flare itself in SLE patients. ${ }^{4}$ A Canadian clinical trial, has recommended vitamin D supplementation in patients with SLE as it showed increased vitamin D levels ameliorated inflammatory and hemostatic markers and showed a tendency toward subsequent clinical improvement. 9 Despite plentiful exposure to sunlight throughout the year in Arabic or Gulf countries, VDD is highly prevalent in Saudi patients with SLE. ${ }^{10}$ But, also in Egyptian patients with SLE. ${ }^{3}$ A recent study from Taiwan has shown that serum vitamin D levels are inversely correlated with SLE disease activity at both active and inactive disease status, as well as, with an active LN disease stage. ${ }^{11}$ On the other hand, association 
of VDD with the development of SLE or certain clinical features or laboratory parameters has been investigated and reported, but the mechanisms behind these associations are still needed to be clarified. Thus, in patients with SLE, VDD is associated with high levels of IL-6 and hematuria. ${ }^{6}$ Furthermore, it also associated with the quality of sleep. ${ }^{12}$ On the contrary, vitamin D intake has been suspected as a risk factor for development of systemic lupus erythematosus in women, however, this has been disproved by Costenbader et al. ${ }^{13}$

In the Kingdom of Bahrain, a population-based study has confirmed a high prevalence of VDD in healthy subjects, especially in females, and that showed a seasonal variation. ${ }^{14}$ Nevertheless, vitamin D status in Bahraini patients with SLE has not been investigated before. In this current study we would like to assess the prevalence of VDD in patients with SLE in Bahrain and to evaluate any association with the clinical predictors in such patients. Our aim is to conduct a retrospective cross-sectional study to investigate the vitamin D serum levels together with other biochemical parameters that generally considered to be involved in VD regulation such as; calcium, phosphorus, and parathyroid hormone (PTH), as well as, alkaline phosphatase as a marker for bone turnover. We aimed to investigate these parameters in a relatively large Bahraini cohort with SLE (58 patients), furthermore, to evaluate the impact of VDD on the exacerbation of pain and ill musculoskeletal health, as well as, clinical data, disease activity as assessed by presence of specific lupus autoantibodies (ds-DNA), but also ANA, C3 and $\mathrm{C} 4$ in these patients group. We also aimed to compare VD serum levels and the other biochemical parameters with the corresponding values in healthy controls.

\section{Material and methods}

\section{Patients}

Fifty-eight Bahraini patients with systemic lupus erythematous (SLE) were included retrospectively in this study. The demographic data are depicted in Table 1. The mean age of the patients was 39.78 years (range 16-61years). However, most of the patients are female 50/58 (86.2\%), while the male patients are only eight (13.8\%). The patients were carefully followed at the rheumatology department at the main hospital in Bahrain "Salmanyia medical complex (SMC)". All patients fulfilled the American College of Rheumatology (ACR) criteria from 1997 for the diagnosis of SLE. ${ }^{15}$ Fifty-eight healthy Bahraini subjects were used as controls. The controls are healthy Bahraini subjects and they are age-match, but only around 74\% (35 females and 8 males) are also sex-matched.

\section{Methods}

The measurement of the serum level of $25(\mathrm{OH})$ Cholecalciferol, vitamin D (D3), was estimated using chemilumenescence immunoassay on Advia Centaur Analyzer (LoD 8.0nmol/L). Serum levels $<30 \mathrm{nmol} / \mathrm{L}$ were considered as VDD, levels between $30 \mathrm{nmol} / \mathrm{L}$ and $50 \mathrm{nmol} / \mathrm{L}(\geq 30<50)$ were classified as vitamin D insufficiency and optimal levels were $\geq 50 \mathrm{nmol} / \mathrm{L}$. The complements, C3 \& C4, were done by automated nephlometry using seimens reagents and $\mathrm{BN}$ Prospect machine. ANA test was done by indirect immunofloresence (IIF) method using hep2 slides from BIORAD Company. Anti-
dsDNA levels were tested by automated ELIA using uniCAP machine from Phadi (pharmacia diagnostics -Thermo scientific co). Calcium, phosphorus and alkaline phosphatase levels in the serum were analyzed using spectrosphometric technique on Advia Chemistry XPT Analyzer. Intact PTH was determined in serum by two-site sandwich immunoassay, using direct chemiluminometric technology on Advia centaur analyzer (analytical sensitivity $0.265 \mathrm{pmol} / \mathrm{L}$ ).

\section{Statistical analysis}

Data was entered and analyzed using SPSS version 23.0. Quantitative variables were presented as mean \pm SD and qualitative variables were parented as count and percentages. Pearson correlation coefficient was used to measure the strength of linear relationship between Vitamin D and the risk factors, while Chi-Square was used to measure the association between Vitamin D deficiency and patient status. Independent 2-sample t-test was used to test the significance difference in population means. P-value $<0.05$ was considered as statistically significant.

\section{Results}

The demographic data of patients and controls is shown in Table 1. The mean age of patients is 39.77 , range between 16-61years (STD 12.98). The mean age for the controls is 44.81 (range 31-55, STD 5.46). Most of our patients are females 50/58 (86\%) and the males are $8(14 \%)$. In the control groups the females are $35 / 58(60 \%)$ and only 23 are males $(40 \%)$. There are statistical differences in patients compared to controls group in each of Phos, ALP, and PTH, but not the calcium. The results of the comparison of vitamin D and other parameters between SLE patients are depicted in Table 2. The mean serum level for vitamin D for SLE patient is 30.67 (range; 11-54nmol/1), while for control the mean serum level of vitamin D is 39.59 (range $13-122 \mathrm{nmol} / \mathrm{l})$. There is statistical significant difference in vitamin D serum levels between SLE patients and controls ( $\mathrm{p}$ value $=0.005$, (95\% CI -15.13- -2.70). (STD 12.11). On classifying the patients into deficient, insufficient and optimal (Figure 1), The current study demonstrated that the deficient SLE patients had lower serum levels compared to deficient control group and that deference was statistical significant ( $\mathrm{p}=>0.01$ ) (Table 3 ).

Regarding, the patient group the deficient were 26(49.1\%), insufficient were $25(47.1 \%)$, while only two patients had optimal levels $(2.8 \%)$. Concerning the controls, deficient were $17(29 \%)$, insufficient were $29(50 \%)$ and optimal were $12(21 \%)$. There is a statistically significant difference between patients and control groups in all risk factors (Phos, PTH and ALP) except Ca with a (p values $\leq 0.001$ in each of the investigated parameters) (Table 2). Table 4 , showed Sperman correlation in patients cohort. There were positive correlations between PTH and Phos, ALP and uric acid $(\mathrm{r}=0.277)$, $(\mathrm{r}=0.275)$ and $(\mathrm{r}=0.584)$, respectively. Also there is correlation between $\mathrm{C} 3$ and $\mathrm{C} 4,(\mathrm{r}=0.609), \mathrm{C} 3$ and CRP $(\mathrm{r}=0.290), \mathrm{C} 3$ and ALP $(\mathrm{r}=0.383)$ and $\mathrm{C} 3$ and Phos $(\mathrm{r}=0.451)$. Table 5, showed the Spearman correlation in the controls group. There are correlation between vitamin $\mathrm{D}$ and $\mathrm{PTH}(\mathrm{r}=0.346)$, but also between $\mathrm{PTH}$ and $\mathrm{Ph}(\mathrm{r}=0.282)$, and between Phos and calcium $(\mathrm{r}=0.282)$. 
Table I Demographic data of the participants

\begin{tabular}{lllllll}
\hline Patcon & Variable & No. (\%) & Minimum & Maximum & Mean & SD \\
\hline \multirow{2}{*}{ Patient(n=58) } & Sex(F) & $50(86.2)$ & 16 & 61 & 39.78 & 12.98 \\
& Age/Year & $8(13.8)$ & & & & \\
Control(n=58) & Sex(F) & $35(60.3)$ & 16 & 61 & 39.78 & 12.98 \\
& Age/Year & $23(39.7)$ & & & \\
\hline
\end{tabular}

Table 2 Comparison of vitamin D, Ca, Phos, PTH and ALP between patients and controls

\begin{tabular}{lllllllll}
\hline & \multicolumn{2}{l}{ Patient*(n=58) } & \multicolumn{2}{l}{ Control(n=58) } & & \multicolumn{2}{l}{ 95\% C.I. } \\
\cline { 2 - 9 } & Mean & SD & Mean & SD & P-Value & Mean diff. & Lower & Upper \\
\hline Vitamin D & 30.67 & 12.11 & 39.6 & 19.68 & 0.005 & -8.92 & -15.13 & -2.71 \\
Ca & 1.95 & 0.69 & 1.99 & 0.36 & 0.723 & -0.04 & -0.24 & 0.17 \\
Phos & 1.06 & 0.48 & 1.3 & 0.23 & 0.001 & -0.25 & -0.39 & -0.11 \\
PTH & 6.34 & 5.65 & 28.87 & 17.01 & $<0.0005$ & -22.53 & -27.23 & -17.84 \\
ALP & 70.78 & 30.42 & 53.72 & 17.23 & $<0.0005$ & 17.05 & 7.93 & 26.17 \\
\hline
\end{tabular}

Table 3 Association between vitamin d deficient class in both patients and controls

\begin{tabular}{lllllll}
\hline & & \multicolumn{2}{c}{ Vitamin D } & & \multirow{2}{*}{ Total } & \multirow{2}{*}{ P- Value } \\
\cline { 3 - 5 } & & Deficient & Insufficient & Optimal & & \\
\cline { 3 - 5 } & & No.(\%) & No.(\%) & No.(\%) & No.(\%) & \\
& Patient & $26(49.1)$ & $25(47.2)$ & $2(3.8)$ & $53(100.0)$ & \multirow{2}{*}{0.01} \\
\multirow{2}{*}{ Patcon } & Control & $17(29.3)$ & $29(50.0)$ & $12(20.7)$ & $58(100.0)$ & \\
& & $43(38.7)$ & $54(48.6)$ & $14(12.6)$ & $111(100.0)$ & \\
\hline
\end{tabular}

Table 4 Pearson correlation coefficient between vitamin D and Ca, Phos, PTH,ALP, C3, C4, ds-DNA, CRP and uric acid in patient

\begin{tabular}{|c|c|c|c|c|c|c|c|c|c|c|c|}
\hline SLE Patients & & $\begin{array}{l}\text { Vitamin } \\
\text { D }\end{array}$ & $\mathrm{Ca}$ & Phos & PTH & ALP & $\mathrm{C} 3$ & $\mathrm{C} 4$ & dsDNA & CRP & $\begin{array}{l}\text { URIC } \\
\text { ACID }\end{array}$ \\
\hline \multirow{2}{*}{ Vitamin D } & Pearson Correlation & 1 & 0.023 & 0.111 & -0.127 & -0.15 & 0.027 & 0.096 & -0.067 & -0.147 & -0.229 \\
\hline & P-Value & & 0.872 & 0.43 & 0.366 & 0.283 & 0.847 & 0.494 & 0.632 & 0.295 & 0.172 \\
\hline \multirow{2}{*}{$\mathrm{Ca}$} & Pearson Correlation & 0.023 & 1 & 0.21 & 0.106 & 0.19 & 0.026 & 0.038 & -0.235 & 0.114 & -0.027 \\
\hline & P-Value & 0.872 & & 0.113 & 0.43 & 0.154 & 0.848 & 0.776 & 0.076 & 0.393 & 0.869 \\
\hline \multirow{2}{*}{ Phos } & Pearson Correlation & -0.111 & 0.21 & 1 & $0.277 *$ & $0.407 * *$ & $0.451 * *$ & 0.204 & 0.143 & 0.16 & 0.18 \\
\hline & P-Value & 0.43 & 0.113 & & 0.035 & 0.002 & 0 & 0.125 & 0.283 & 0.231 & 0.265 \\
\hline \multirow{2}{*}{ PTH } & Pearson Correlation & -0.127 & 0.106 & $0.277 *$ & 1 & $0.275^{*}$ & 0.134 & 0.161 & 0.203 & -0.046 & $0.584 * *$ \\
\hline & P-Value & 0.366 & 0.43 & 0.035 & & 0.037 & 0.316 & 0.226 & 0.127 & 0.729 & 0 \\
\hline \multirow{2}{*}{ ALP } & Pearson Correlation & -0.15 & 0.19 & $0.407 * *$ & $0.275 *$ & 1 & $0.383 * *$ & 0.163 & 0.114 & 0.205 & 0.16 \\
\hline & P-Value & 0.283 & 0.154 & 0.002 & 0.037 & & 0.003 & 0.221 & 0.395 & 0.122 & 0.324 \\
\hline \multirow{2}{*}{$\mathrm{C} 3$} & Pearson Correlation & 0.027 & 0.026 & $0.451 * *$ & 0.134 & $0.383 * *$ & 1 & $0.609 * *$ & -0.036 & $0.326^{*}$ & 0.08 \\
\hline & P-Value & 0.847 & 0.848 & 0 & 0.316 & 0.003 & & 0 & 0.789 & 0.012 & 0.623 \\
\hline \multirow{2}{*}{$\mathrm{C} 4$} & Pearson Correlation & 0.096 & 0.038 & 0.204 & 0.161 & 0.163 & $0.609 * *$ & 1 & -0.023 & $0.290 *$ & 0.249 \\
\hline & P-Value & 0.494 & 0.776 & 0.125 & 0.226 & 0.221 & 0 & & 0.863 & 0.027 & 0.121 \\
\hline \multirow{2}{*}{ ds-DNA } & Pearson Correlation & -0.067 & -0.235 & 0.143 & 0.203 & 0.114 & -0.036 & -0.023 & 1 & -0.016 & 0.253 \\
\hline & P-Value & 0.632 & 0.076 & 0.283 & 0.127 & 0.395 & 0.789 & 0.863 & & 0.907 & 0.115 \\
\hline \multirow{2}{*}{ CRP } & Pearson Correlation & -0.147 & 0.114 & 0.16 & -0.046 & 0.205 & $0.326 *$ & $0.290 *$ & -0.016 & 1 & -0.066 \\
\hline & P-Value & 0.295 & 0.393 & 0.231 & 0.729 & 0.122 & 0.012 & 0.027 & 0.907 & & 0.688 \\
\hline \multirow{2}{*}{ URICACID } & Pearson Correlation & -0.229 & -0.027 & 0.18 & $0.584 * *$ & 0.16 & 0.08 & 0.249 & 0.253 & -0.066 & 1 \\
\hline & P-Value & 0.172 & 0.869 & 0.265 & 0 & 0.324 & 0.623 & 0.121 & 0.115 & 0.688 & \\
\hline
\end{tabular}

Citation: Farid E, Hassan AB, Jaradat AA, et al. Prevalence of vitamin D deficiency in adult patients with systemic lupus erythematosus in Kingdom of Bahrain. MOJWomens Health. 20I7;6(I):338-342. DOI: I0.15406/mojwh.2017.06.00I47 
Table 5 Pearson correlation coefficient between vitamin D and Ca, Phos, ALP and PTH in Controls

\begin{tabular}{|c|c|c|c|c|c|c|}
\hline \multicolumn{2}{|c|}{ Control Group } & \multirow{2}{*}{$\begin{array}{l}\text { Vitamin D } \\
1\end{array}$} & \multirow{2}{*}{$\begin{array}{l}\mathbf{C a} \\
-0.054\end{array}$} & \multirow{2}{*}{$\begin{array}{l}\mathbf{P h} \\
0.099\end{array}$} & \multirow{2}{*}{$\begin{array}{l}\text { ALP } \\
-0.036\end{array}$} & \multirow{2}{*}{$\begin{array}{l}\text { PTH } \\
0.346 * *\end{array}$} \\
\hline Vitamin D & Pearson Correlation & & & & & \\
\hline vilant & P-Value & & 0.688 & 0.461 & 0.787 & 0.008 \\
\hline \multirow{2}{*}{$\mathrm{Ca}$} & Pearson Correlation & -0.054 & 1 & $0.650 * *$ & 0.112 & 0.06 \\
\hline & P-Value & 0.688 & & 0 & 0.403 & 0.657 \\
\hline \multirow{2}{*}{ Phos } & Pearson Correlation & 0.099 & $0.650 * *$ & 1 & 0.165 & $0.282 *$ \\
\hline & P-Value & 0.461 & 0 & & 0.216 & 0.032 \\
\hline \multirow{2}{*}{ ALP } & Pearson Correlation & -0.036 & 0.112 & 0.165 & 1 & -0.121 \\
\hline & P-Value & 0.787 & 0.403 & 0.216 & & 0.365 \\
\hline \multirow{2}{*}{ PTH } & Pearson Correlation & $0.346 * *$ & 0.06 & $0.282 *$ & -0.121 & 1 \\
\hline & P-Value & 0.008 & 0.657 & 0.032 & 0.365 & \\
\hline
\end{tabular}

\section{Classification of vitamin D in control Group}

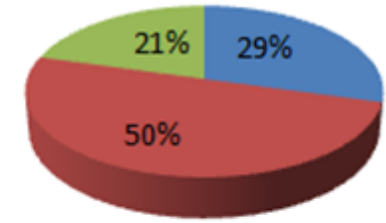

\section{Classification of vitamin D in SLE Patients}

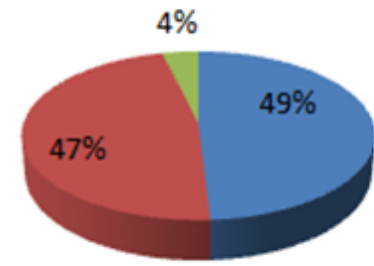

Deficient

- insufficient

Optimal

Figure I Classification OfVitamin D in Patients vs Controls.

\section{Discussion}

The current retrospective study is a cross-sectional study of a large cohort of Bahraini SLE patient and is the first study to investigate the vitamin D status in Bahraini SLE patients. We found that there is a high prevalence of VDD among Bahraini SLE patients. Almost half of our patients $(49.1 \%)$ having vitamin deficiency with a level less than $30 \mathrm{nmol} / \mathrm{L}$, while $96 \%$ of the patients have abnormal vitamin D levels (deficient or insufficient), this is in keeping with other studies in other parts of the world. ${ }^{16}$ In a Norway study the majority of recently settled immigrants from all age groups from the Middle East, South Asia and Africa showed that female adolescents from these regions had low levels of $25(\mathrm{OH})$ D. ${ }^{17,18}$ It is important to note that even when patients were classified according to their vitamin D into three levels, we could still be able to show statistically significant difference in VDD class (vitamin D serum levels $<30 \mathrm{nmol} / \mathrm{l}$ ) between patients and controls. Our finding about high prevalence of vitamin D is consistent with another study in the region that has revealed VDD in Saudi patients with SLE. ${ }^{10}$

Surprisingly, our study failed to describe any correlation between hypovitaminosis and the risk factors for disease activity as assessed by the presence of specific lupus autoantibodies (ds-DNA) and/or low $\mathrm{C} 3$ and $\mathrm{C} 4$, or between hypovitaminosis and any of the investigated parameters including the risk factors for vitamin D disregulation in these patients group. These findings needed to be confirmed by another Bahraini study using any of the standard disease activity indices use for SLE disease. In contrast, in the control group the current study revealed a positive correlation between vitamin $\mathrm{D}$ and $\mathrm{PTH}$ at 0.01 and between PTH and Phos at 0.05, however, a correlation between Ca and Phos at 0.01 was also found. ${ }^{19}$ The significance of low Vitamin D in our patients with SLE may be explained partly by the state of chronic inflammatory condition and renal involvements as many of our SLE patients have glomerular disorders (data not shown). It will be interesting to evaluate the effect of correcting hypovitaminosis $\mathrm{D}$ in these patients cohort and observing the frequency or severity of arthralgia and myalgia and also if there will be any association with any of disease activity indices. In conclusion, the current results about high prevalence of VDD suggest VDD as a possible risk factor for SLE in Bahrain and provide guidance for future studies looking at a potential role of vitamin D in the prevention and/or treatment of SLE. 
The present study also highlights the need for another study looking 9 . for the effects of correcting hypovitaminosis on the clinical status and disease activity in Bahraini patients with SLE disease.

\section{Acknowledgements}

None.

\section{Conflict of interest}

The author declares no conflict of interest

\section{References}

1. Hoffecker BM, Raffield LM, Kamen DL, et al. Systemic lupus erythematosus and vitamin D deficiency are associated with shorter telomere length among African Americans: a case-control study. PLoS One. 2013;8(5):e63725.

2. Kamen DL, Cooper GS, Bouali H, et al. Vitamin D deficiency in systemic lupus erythematosus. Autoimmun Rev. 2006;5(2):114-117.

3. Hamza RT, Awwad KS, Ali MK, et al. Reduced serum concentrations of 25-hydroxy vitamin $\mathrm{D}$ in Egyptian patients with systemic lupus erythematosus: relation to disease activity. Med Sci Monit. 2011;17(12):CR711-718.

4. Dall'Ara F, Andreoli L, Piva N, et al. Winter lupus flares are associated with low vitamin D levels in a retrospective longitudinal study of Italian adult patients. Clin Exp Rheumatol. 2015;33(2):153-158.

5. Bogaczewicz J, Sysa-Jedrzejowska A, Arkuszewska C, et al. Vitamin D status in systemic lupus erythematosus patients and its association with selected clinical and laboratory parameters. Lupus. 2012;21(5):477-484.

6. de Souza VA, Bastos MG, Fernandes NM, et al. Association of hypovitaminosis D with Systemic Lupus Erythematosus and inflammation. J Bras Nefrol. 2014;36(4):430-436.

7. Kamen DL. Environmental influences on systemic lupus erythematosus expression. Rheum Dis Clin North Am. 2014;40(3):401-12.

8. Jacobs J, Korswagen LA, Schilder AM, et al. Six-year follow-up study of bone mineral density in patients with systemic lupus erythematosus. Osteoporos Int. 2013;24(6):1827-1833.
Abou-Raya A, Abou-Raya S, Helmii M. The effect of vitamin D supplementation on inflammatory and hemostatic markers and disease activity in patients with systemic lupus erythematosus: a randomized placebo-controlled trial. J Rheumatol. 2013;40(3):265-272.

10. Damanhouri LH. Vitamin D deficiency in Saudi patients with systemic lupus erythematosus. Saudi Med J. 2009;30(10):1291-1295.

11. Lin TC, Wu JY, Kuo ML, et al. Correlation between disease activity of pediatric-onset systemic lupus erythematosus and level of vitamin D in Taiwan: A case-cohort study. J Microbiol Immunol Infect. 2016;S16841182(16):00006-00012.

12. Gholamrezaei A, Bonakdar ZS, Mirbagher L, et al. Sleep disorders in systemic lupus erythematosus. Does vitamin D play a role? Lupus. 2014;23(10):1054-1058.

13. Costenbader KH, Feskanich D, Holmes M, et al. Vitamin D intake and risks of systemic lupus erythematosus and rheumatoid arthritis in women. Ann Rheum Dis. 2008;67(4):530-535.

14. Golbahar J, Al-Saffar N, Altayab Diab D, et al. Predictors of vitamin D deficiency and insufficiency in adult Bahrainis: a cross-sectional study. Public Health Nutr. 2014;17(4):732-738.

15. Hochberg MC. Updating the American College of Rheumatology revised criteria for the classification of systemic lupus erythematosus. Arthritis Rheum. 1997;40(9):1725.

16. Mok CC. Vitamin D and systemic lupus erythematosus: an update. Expert Rev Clin Immunol. 2013;9(5):453-463.

17. Eggemoen AR, Knutsen KV, Dalen I, et al. Vitamin D status in recently arrived immigrants from Africa and Asia: a cross-sectional study from Norway of children, adolescents and adults. BMJ Open. 2013;3(10):e003293.

18. Knutsen KV, Brekke M, Gjelstad S, et al. Vitamin D status in patients with musculoskeletal pain, fatigue and headache: a cross-sectional descriptive study in a multi-ethnic general practice in Norway. Scand J Prim Health Care. 2010;28(3):166-171.

19. Peracchi OA, Terreri MT, Munekata RV, et al. Low serum concentrations of 25-hydroxyvitamin D in children and adolescents with systemic lupus erythematosus. Braz J Med Biol Res. 2014;47(8):721-726. 